\title{
The Role of the Social Worker in Modifying the Process of Decision Making through using Cognitive Behavioral Therapy
}

Mrs. Mariam Alnuaimi

Social Wellbeing Department, Collage of Humanities and Social Sciences, United Arab Emirates University, UAE

Corresponding Author: Mrs. Mariam Alnuaimi, E-mail: 201401123@uaeu.ac.ae

\section{ARTICLE INFORMATION}

Received: August 21, 2020

Accepted: September 25, 2020

Volume: 2

Issue: 5

\section{KEYWORDS}

Cognitive-behavioural therapy, Decision making, Social Worker, Techniques

\section{ABSTRACT}

The idea of cognitive-behavioural therapy is helping to improve the way of thinking or emotions to see the result on the client's behaviour. Some of the clients find it difficult to change the process of thinking when they are trying to take a step of changing. In this article, I will provide brief information on the role of the social worker in modifying the process of decision making by using cognitive behavioural therapy. Some research studies mentioned the importance of $\mathrm{CBT}$ and the CBT using in the decision-making process. Also, the several cognitive techniques that might benefit the social workers or the therapists on applying into the sessions or psychotherapy.

\section{Introduction}

The cognitive-behavioural therapy is one of the most and effective treatment using by the social workers, psychologists or other therapists who are dealing with human specifically. Moreover, the CBT is a treatment result from the integration from behaviour, thinking and emotions were the social workers, or the therapists is dealing with many clients who suffer from the cognition distortions and make wrong decisions or an unconscious and thoughtful decisions so that it will affect their life in future and might lose them the control of completing their life. On my topic, I am trying to figure the role of the social worker while using the cognitive behavioural therapy by modifying the process of client's decision making in order to suggest for the therapists uses certain ways of cognitive strategies to achieve the required objectives of the intervention process. The aim of this paper, to cover the lack of the limited research of guiding the therapist on the process of modifying decision making.

\section{Literature Review}

In the research named is (the key principles of cognitive-behavioural therapy). The researchers have turned their focus towards the key principles, which are associated with cognitive behavioural therapy (CBT). It is vital to know that patients with mental illness are treated with different kinds of methods, and cognitive behavioural therapy is one of the effective methods used by professionals. It has been an effective method to treat people who are dealing with various kinds of mental disorders. The researchers have analyzed each aspect of the cognitive behavioural therapy to see how it works, what its techniques are, and how effective it has been over the years. It is important to mention here that cognitive behavioural therapy is one of the psychotherapeutic techniques, which has been researched with a great amount, and a lot of empirical evidence has been found to know that cognitive behavioural therapy is one of the best methods to use for mentally ill people.

When the core of these methods is reviewed, it is evident that CBT is having its basis on a cognitive model, which tends to deal with mental illness. The primary idea behind developing this method is that the behaviour and emotion of people are influenced and driven by their perceptions, which they develop about certain events. It means that the actual situation may be different, but it is the interpretation of a person, who is making it a troublesome situation for him/her. One good example of this expression is the negativity of depressed patients. It is a fact that when a person is depressed, he/she sees negativity

\footnotetext{
K C AL-KINDI CENTER

R D FOR RESEARCH AND $\mathbf{R} \mathbf{D}$ DeVELOPMENT
} Your gateway to world-class research
Published by Al-Kindi Center for Research and Development. Copyright (c) the author(s). This is an open access article under CC BY license (https://creativecommons.org/licenses/by/4.0/) 
in everything, whereas some situations may be actually positive. The researchers have identified three levels, which are associated with the cognition of a person; first is his/her core beliefs, the second is the negative thoughts, and the third one is dysfunctional assumptions, and all these three levels develop a model of cognition for a person.

So, the scientists have developed CBT to deal with this cognition, which is going in the wrong direction. The researchers also focused on some of the techniques, which are used in CBT. The first technique is called cognitive technique, which is used to understand the thought process of an individual, and then make the required changes. The second technique in CBT is called the behavioural technique. In this technique, different activities are scheduled for individuals to make positive changes in their behaviour. The article also included the evidence, which showed that CBT is one of the most effective techniques. The research has found that people dealing with depression can get better when they are treated with CBT. The various empirical research studies have proved that $C B T$ is quite beneficial in treating patients with a variety of mental illnesses such as anxiety disorders, childhood depression, post-traumatic stress disorder, social phobia, panic disorder, as well as, bipolar disorder. It was also explained that CBT comes with so many benefits like it is not only a cost-effective method, but it also comes with long-term positive outcomes. The use of CBT has also been allowed in primary care with certain conditions (Fenn \& Byrne, 2013).

Decision making is an important trait or ability for any individual to have because one has to make such decisions in life. The authors in this research (The Effect of Cognitive Behavior Therapy on Decision Making in Adolescents who Self-Harm: A Pilot Study) have focused on the element of decision making with regards to adolescents, who are involved in the practice of selfharm. The basic purpose of this pilot study is to measure the effects made by cognitive behavioural therapy on these adolescents. It has been evident in the research that adolescents, who get involved in self-harm, they actually make this mistake because they are not good in their decision-making ability. Some of them don't have any ability to make proper decisions regarding their lives, and some others have a fear of decision in their minds. It has been observed that when adolescents deal with any kind of emotional and behavioural issues, they get involved in self-harm, which also leads to committing suicide. There could be many reasons behind the emotional and behavioural issues faced by these adolescents; however, if they could have better decision-making ability, then they can deal with such issues in an effective manner rather than getting involved in self-harm.

So, the purpose of this pilot study was to investigate, whether CBT can help adolescents in better decision making, who have committed self-harm of any kind. It was analyzed how decision-making changes for those who committed self-harm and received CBT sessions. The participants of the study were taken from the Community Child and Adolescent Mental Health Service (CAMHS). It is important to mention here that personal contacts and schools were also used to get participants. The age range of the participants in the study was 12-18 years old. The written consent was taken from the parents of the children, who were under the age of 16 . The participants were screened by giving them depression and IQ related questions. Three groups were formed for the participants, categorizing on the basis of self-harm, which they committed. The adolescents with self-harm were given psychological treatment with the help of 12 sessions under the essence of CBT.

After applying the methodology, and implementing CBT sessions on adolescents, when results of the study were compiled, it was found that if adolescents do not receive any kind of psychological treatment or intervention, who has been involved in self-harm: they tend to become worst in their decision making with the passage of time. It was also found that adolescents who received sessions of CBT to get treatment for their psychological and emotional problems; they showed a lot of improvement in their decision-making abilities. It was also suggested by the study that special kind of CBT sessions should be developed for adolescents with a history of self-harm so that they can be treated to get better in the future. The findings made by this study are very much encouraging for future research because it has provided a future direction regardless of its flaws, and researchers can look deeper into this issue to find how CBT can be used more effectively to improve the decisionmaking ability of adolescents. It can really become a huge step for adolescents and their well-being because when they make better decisions, they will refrain from self-harm and problematic behaviours (OLDERSHAW, et al., 2012).

Another article of Cognitive behavioural therapy: why primary care should have it all, the researchers have not focused on the efficacy of cognitive-behavioural therapy in general terms; rather they have tried to discuss why primary care should adopt the essence of cognitive-behavioural therapy. It is described in the article that CBT is one of the short-term treatment methods, which is used to make changes in the unhelpful thought process and behaviour of individuals, who are dealing with any kind of mental disorder or issue. It is important to know that CBT has been recommended by the National Institute for Health and Clinical Excellence (NICE) of the UK. They have recommended using it for treating a variety of mental health problems such as anxiety, bulimia nervosa, obsessive-compulsive disorder, post-traumatic stress disorder, and depression. Another great thing about CBT is its ability to handle disorders related to sleep. Sleeping disorders can play a part in mental and physical health problems such as fatigue, pain, and phobias. 
The researchers have briefly analyzed the benefits of $C B T$ for different purposes so that they can lead the discussion towards primary care. It was explained that there are so many methods to deliver sessions of CBT for people from different age range, such as kids, young adults, older adults, etc. The use of CBT is evident in secondary care, and its use for primary care has also been observed. However, the use of CBT in primary care has not been gone to its full potential. It is mentioned that primary care settings can have so many conditions to deal with, but CBT is primarily used to deal with an issue related to anxiety and depression. The fact of the matter is that CBT can be used for various other primary care conditions so that it can bring more benefits for the mentally ill patients. The evidence is there that the use of CBT in primary care is gradually increasing, but still; it is not up to the required level.

There are certain barriers, which are making obstacles for the CBT to be fully used in the primary care setting. One of the biggest issues in this regard is that professional staff for CBT is not sufficient. There is a huge shortage of staff to perform relevant duties in primary care. The number of Clinical psychologists is very limited as compared to any other medical field. That's why; the constant delivery of CBT in primary care is not practically possible. It was found that individuals come up with more complex mental problems as compared to just depression, and they need immediate help from the primary care system to get treatment. The professional staff should be increased with the passage of time, and resources should be developed so that patients can be treated in primary care settings without wasting any time. So, it is necessary to make sure that CBT is widely spread in the setting of primary care with all its capability and potential (Blane, Williams, Morrison, Wilson, \& Mercer, 2013)

The most effective one is (Cognitive-behavioral therapy and decision science) and in the field of psychological science, decision science (DS) and Cognitive-behavioral therapy (CBT) has been emerging for more research and analysis to get more insights for both. That's why the researchers of this study have turned their focus to CBT and DS. It is important to keep in mind that both these fields are extremely different areas to conduct studies. However, it is a fact that both are somewhat related to each other on the basis of human behaviour, cognition, and information processing. The basic purpose of this research study is to analyze both decision science and CBT with all their aspects so that they are reviewed with their differences, as well as similarities. It is also reviewed how future research can help to identify patterns for both fields so that both can play a vital role in informing each other. The research was conducted with an expectation that it would help to make improvements for both fields. The application of CBT is made to address a range of concerns associated with mental illness regarding the behaviour and cognition of patients. On the other hand, the role of decision science has been critical in areas of economics and medicine. The patients who deal with a variety of mental health issues, they are treated with the help of CBT. A variety of techniques are used in this regard, such as goal setting, exposure, relapse prevention, self-monitoring, problemsolving, cognitive-behavioural assessment, and behavioural experiments.

On the other hand, decision science is an area of study, which observes how people tend to make decisions when they deal with different and diverse settings. It is said that the cognitive abilities of humans are somewhat limited, and that's why humans are not able to use it to full capacity to get success. At this point of cognitive bound, people can't search more to find more opportunities. Once both concepts were fully explained in the article, the researchers reviewed them by making a detailed comparison of both. After analyzing and comparing both, it was found that both fields do have the potential and capability to support, inform, as well as, help each other to build upon with more aspects. It was also found that there is a possibility that both DS and CBT can be used with the integration, which can bring more benefits for both fields. So, it was recommended that more research should be conducted in this direction to find out more ways, how DS and CBT can be integrated, and how both can help each other to bring more efficacy and effectiveness for the people. That's why it is vital to make further investigation in this direction (Leddy, Anderson, \& Schulkin, 2013).

\section{Cognitive role of the therapist}

The therapists playing many roles in modifying decision making with their clients in order to use different strategies such as cognitive strategies and behavioural strategies to achieve the outcomes of cognitive-behavioural therapy. We will discuss how the therapists use a specific strategy, intervene by asking questions and which homework is applicable for a certain strategy.

\section{Related Cognitive techniques of $\mathrm{CBT}$ in modifying decision making with clients}

\subsection{Identifying the Short-Term and Long-Term Goals Technique}

There are two types of clients who set their goals based on the efforts they done toward a particular action. Such as, clients who focused on short term goals they are immediately taking a decision based on their goals setting and since the client thinks with this way; he immediately thinks about the quick consequences and fail to realize the importance of long-term gains. Unlike the clients who see the goals with long-term is more important and come up with better outcomes. Most of the 
long-term goals connected to something we feel it important to our life such as health, education, financial well-being, occupational and social relationships while the short-term goals focus on instant gratification such as food, shelter and entertainment (Leahy, 2020).

The role of the therapist can give the client task on how to differentiate between setting long-term goals and setting short term-goals especially with the sensitive goals that are concentrated of well-begin as physical appearance, intimate relationships or efficiency at work. The therapist can ask the client to think about focusing on the short-term and long-term goals in which issues can create. As well as, the therapist can only focus in long-term goals and set both a current plan by how might the client achieve the long-term goals, and the therapist can let the client express the future feelings when he reaches what he wants (Robert, 1997).

\subsection{Pre-commitment Strategies}

The easiest way to define this strategy that when you make a decision toward something, and you be a commitment to it before you really engage in real life. Some clients put a list of punishment if they go against what they decided before. Such as, fatty clients may feel upset when they eat more sugar and sweets; so, they resist to eat in order to look in a healthy way and be more fit since they believe this way, they might avoid buying sugar products or may having a membership at the gym which allow them to obligate toward their long-term goals.

The role of the therapist might come in many ways when using this technique; they can ask their client's different questions to solve this problem in the future by setting a plan on making a decision now. Clients who have a problem in spending more money and they face a financial problem monthly; client and the therapist can set a plan by writing the thing that needs to change, the advantages of changing this behaviour and steps to help for succus such as saving the amount of salary in different account in the bank. This can help the client to see whether he can be a commitment to the achieve this goal and insistence towards its implementation (Leahy, 2020).

\subsection{Modifying Emotional Reasoning in Decision Making Technique}

A key component of problematic decision-making is relying on emotions to determine risks, consequences, and alternatives. This same as affective inferences, so when people are depressed or anxious, they mostly used their emotions to predict the outcome. For example, people who afraid of the airplane and they feel something bad will happen to his/her flight; this is because of negative feelings on behalf of travelling by plane. In general, if the person is in a bad mood highly likely will avoid the risky or situations that seem difficult. However, if the person feels happy and bright mostly will face any challenges, and the less to avoid risky events.

The role of the therapist while using this strategy is to encourage the client to think about the previous, and current decision that causes issues, after one can think about the negative emotions that links with the decision and try to give a decision can result from negative emotion. Also, the therapist can give the client task to think about the same situation if he is happy and optimistic and how the client will think and decide based on his emotional state. This might help the client on thinking about his/her mood state to decide in a positive and healthy way (Leahy, 2020).

\subsection{Reframing Change as Gain Rather Than Loss Technique}

Most clients find difficulties on see the positive part through tragedy and properly see the negative part they lost at the end such as thinking about bad feelings come with the event, bad mood and psychological changes or financial losses that may produce. The clients have a fear of something that will change in the future with big losses and forget about the gain will come with also they turn down opportunities that could be rewarding to avoid the risk of losing something else that feels good enough; therefore, they will make irrational decisions every day simply to avoid losing.

The role of the therapist can come as, asking the client question if it is possible that when you think about making a decision, you focus mainly on the downside or the loss part while ignoring the things that might be gained? In addition, the therapist can create a question as what will happen if the client focusing at a loss so the client can tell about the real loss as the woman who thinks about the divorce, she might answer the question as losing the attachment with this person, feeling lonely, lose joy and happy memories carries by a lost person. Whereas the second question can come as what will happen if the client focusing at gain to tell the story of the woman can stop feeling low self-esteem, no one can abuse her, and all these feelings toward her lost may change to gain when she feels something will change to the better. The idea of using this strategy is the goal to experience a new way of thinking by see the more precious rather than see as pointless while you experience the same situation (Leahy, 2020). 


\subsection{Overcoming Regret Avoidance Technique}

People try to avoid the decisions that might allow them to feel extremely bad about the future, which may get a negative result and bad emotions because of desiring to change. For example, the decision that is making the client change his/her job to another job, the client who has been in an experience of changing crucial decisions can result with unseen problems at this time. This is mean the client will be afraid to take individually serious decisions because of the similar experience happen to $\mathrm{him} /$ her or someone related, this brought a lot of extreme negative emotions toward changing. The client feels safe when a group of people fail to with himself/herself, and that's we could it call it projection which an individual attributes unwanted thoughts, feelings and motives onto another person or group. Moreover, avoiding regret may produce the inability to make decisions at all and regret not always lead to rumination -deep considering of thoughts- such as saying "I am always regret doing this".

The role of the social worker may come as asking the client to mention the past and previous decisions that were difficult to make, how much the client feels guilty for a taking the past decision and fail during that period and is the client focusing in regret long term or short term (Leahy, 2020).

\subsection{The decision for a Future Self Technique}

One of the main problem considerations on making decisions is preferring the small rewards that come in a short time rather than taking seriously the greatest rewards that come after a period of time. It seems theses clients make decisions based on the feelings on how to get something immediately rather than wait to get something even if it values less. Consequently, some clients cannot imagine a positive future self because they feel in a negative area, surrounded them and took of them a lot of advantages. For example, the client can think with a decision after graduating from high school by completing an undergraduate degree or looking for a job to earn money. Some clients see the decision of the job is easier, quicker than studying five years without having a source of income. On the other hand, the decision of the job might be randomly or not specialized in a specific area and less salary than a higher degree holder.

The role of the therapist may let the client thinks about the way of talking with himself/herself at this moment and the way of talking after 20 minutes. This exercise can train the client to avoid taking immediate decisions with fewer features; since you have the opportunity for an adventurous decision with more features. On the other word, the client can set the decision that needs to think about himself/herself of now, after one year, after ten years and how much this decision will affect yourself after a period of time. If the decision seems to be risky one or more years, the client needs to change the way of thinking now to avoid something bad happen in the future (Leahy, 2020).

\subsection{Diversifying Sources of Reward Technique}

When clients have many sources of rewarding in the future, they become more flexible in taking and thinking of making decisions. Also, it can allow them to be capable of practicing various positive activities in future (Leahy, Holland, \& McGnii, 2012). But the cognitive problems might happen with the person who is ready to take risks to get rewards. Therefore, they put "should" statement if the event has been a success and there are many positive things get prove while this will be ignored if the statement took an opposite direction which will make the client think of doubts about its capabilities or permanent failure. In this case, the therapist may ask the client which is the interesting things can practice were allow him/her of having the rewarding whenever he/she reach an important milestone or achieve a specific goal.

\subsection{Comparing Risk versus Risk Technique}

In the overall of taking decisions is when the client has a rational view by the balance between the understandable risks versus incomprehensible risks. Each risk the client confused to take; it has chance whether the client is losing or earning the opportunity. However, most of the clients who find difficulties in balancing things together, highly likely they lost many chances that change their life for many years. The clients thought there are a lot of risks that need to avoid, which makes them feel fear to make decisions, and that is the reason for making them avoid risks. For example, the student in the university who is studying at the major because his/her friend entering first and he/she wants to join with the friend, but after a long period of time, the student realize he/she is not interested in studying this major, and he/she thinks to change to another one, but it is not easy to change the undergraduate major because the student thinks about the years that spending on studying things didn't like, and how much time should he wait to be accepted on the new one, he/she counts off the graduation, but in the same time he/she believes of the degree will not help them on getting a satisfying job. As this cognitive conflict comes to the client, he might end the studying without the feeling of the passion that every student feels and lives (Leahy, 2020). 
In this case, the therapist may help the client on explaining that everything might have risk and opportunity to lose or fail. So, the idea is to think in a way, making you feel the risk is less with high expectations of winning in the chance. Another way may come as the client thinking on the opportunity may lose when you take a decision of taking it or ignoring the decision.

\section{Discussion}

We propose that since social workers constitute not just the biggest group of psychological health providers but also the biggest group of providers of emotional well-being administrations to minorities that are underrepresented in research, we are interestingly situated to advance and direct clinical research with these populaces. This would take into account the advancement of more viable culture-sensitive treatment interventions and further reinforce the role of social work in the process of decision making by using cognitive behavioural therapy. There is a need for extensive research on the part of the social worker in modifying the process of decision making through using cognitive behavioral therapy as currently there are not enough literature and evidence on the situation. Increment in the research of CBT as a decision-making process will not only help therapists in applying CBT process or make progress of client's situation but it will also increase the knowledge poll. We suggest more research is needed to be done in this area for educational and treatment purposes.

\section{Conclusion}

To conclude, there are many ways of modifying the process of decision making by using CBT strategies. The social worker should look for the ways that are suitable for the client and tries to the best strategy for the benefit of the client as it is trying to improve this area of thinking although the hardest work should be done to explore the client's past, and experience led it to think this way. Decision making one of the complex processes has to work with the client's short and long term. Also, there are gaps facing to complete this research and one of them; limited researches study the decision-making process which guides the social worker on working with the client through sessions. Thus, we need to step up our efforts to understand the deep relationship between these two concepts and try come up together by using different ways to understand the client's thinking and experience in making decisions.

\section{References}

[1] Blane, D., Williams, C., Morrison, J., Wilson, A., \& Mercer, S. (2013). Cognitive behavioural therapy: why primary care should have it all. British Journal of General Practice, 63(607), 103-104.

[2] Fenn, K., \& Byrne, D. (2013). The key principles of cognitive behavioural therapy. InnovAiT: Education and inspiration for general practice, 6(9), 579-585.

[3] Leahy, R. (2020). Cognitive Therapy Techniques a Practitioner's Guide. Dar Alfiker for publishing \& distributing, Aman.

[4] Leahy, R. L., Holland, S. J., \& McGnii, L. K. (2012). Treatment plans and interventions for depression and anxiety disorders.

[5] Leddy, M. A., Anderson, B. L., \& Schulkin, J. (2013). Cognitive-behavioral therapy and decision science. New Ideas in Psychology, 31, 173-183.

[6] OLDERSHAW, A., SIMIC, M., GRIMA, E., JOLLANT, F., RICHARDS, C., TAYLOR, L., \& SCHMIDT, U. (2012). The Effect of Cognitive Behavior Therapy on Decision Making in Adolescents who Self-Harm: A Pilot Study. Suicide and Life-Threatening Behavior, 42(3).

[7] Robert, L. (1997). An investment model of depressive resistance. Journal of Cognitive psychotherapy, 3-9. 